\title{
Introduction to the Reproducible Open Coding Kit (ROCK)
}

Gjalt-Jorn Ygram Peters \& Szilvia Zörgő

\section{IMPORTANT NOTICE}

This document has now been superseded by the ROCK Book, and Open Access book that is available at https://rockbook.org. We leave it here for consistency of the record, but all content in this document has been (or will be) migrated to the ROCK book, as well as a lot more.

This document is the full text of "Introduction to the Reproducible Open Coding Kit (ROCK)", a manuscript that is based on a vignette that accompanies the rock $\mathrm{R}$ package.

Please cite this document as (note that the most current version of this manuscript is hosted at PsyArXiv at https://psyarxiv.com/stcx9 - please check that URL before citing this article):

Peters, G.-J. Y., \& Zörgő, S. (2019). Introduction to the Reproducible Open Coding Kit (ROCK). PsyArXiv. doi:10.31234/osf.io/stcx9

The documentation for the $\mathrm{R}$ functions presented in this manuscript, is available at the package's pkgdown website at r-packages.gitlab.io/rock.
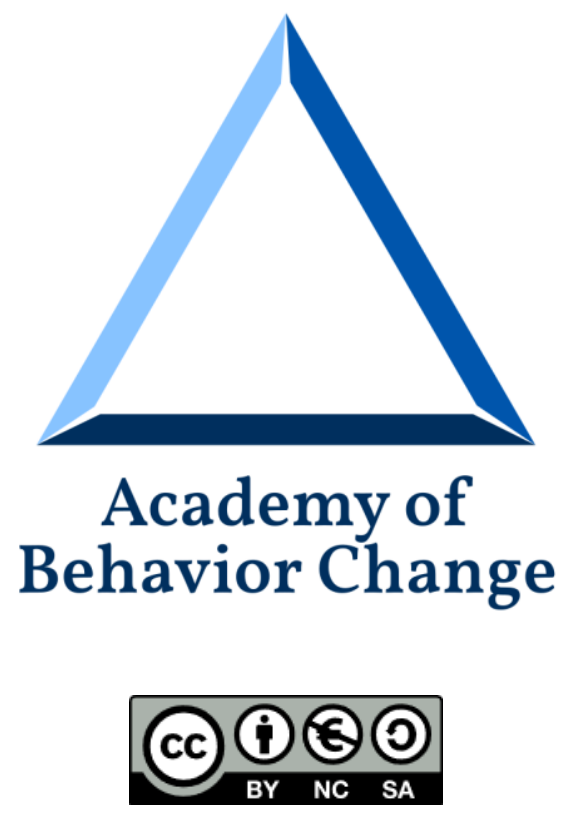
SOME LINKS RELATED TO THE ROCK

R package 'rock'

https://gitlab.com/r-packages/rock

ROCK tutorial

https://sci-ops.gitlab.io/rock-tutorial/

The ROCK Book

https://rockbook.org

iROCK

https://i.rock.science

Gitlab repository for ROCK

https://r-packages.gitlab.io/rock

Prezi introducing you to the ROCK

https://osf.io/nu3mg/ 